\title{
UTILIZAÇÃO DE FERRAMENTAS AUDIOVISUAIS PARA EDUCAÇÃO EM SAÚDE NA ATENÇÃO PRIMÁRIA.
}

\author{
USE OF AUDIOVISUAL TOOLS FOR HEALTH EDUCATION IN PRIMARY CARE. \\ Antonio Rafael da Silva ${ }^{1}$, Hyllary Silva Mota ${ }^{1}$, Kallyny Marques Linhares ${ }^{1}$, Raquel Lino de \\ Menezes ${ }^{1}$, Taisa Freire Mororó de Sá ${ }^{2}$, Carla Jordana de Oliveira Nascimento ${ }^{1}$ \\ Universidade Regional do Cariri-URCA ${ }^{1}$, Escola de Saúde Pública do Ceará ${ }^{2}$
}

\section{Abstract}

Health education is indispensable for SUS, in care and in health prevention and promotion actions. The family health strategy is important for the practice of educational actions, given the proximity to the community. The use of information and communication technologies in health has been expanded in recent years, with the insertion of tools that streamline the flow of information, such as social media. The objective is to highlight the use of technological tools as a support for work processes in primary health care. Descriptive and experience report, part of the perceptions and experiences in health education in primary care through audiovisual tools. The projects called: "ConexãoSaúde" and "Hora da Saúde", were used to bring information to the community about health through audio and videos. With the COVID-19 pandemic, primary health care had to adopt strategic measures to continue offering health actions, as rounds of talks, lectures and events in the basic health units were not being carried out. It was observed that technological resources are of great importance for health education, allowing collective learning and greater interaction between the health team and the community.

Keywords: Health education.Primary Health Care.Access to Essential Medicines and Health Technologies. Coronavirus infections.
Resumo

A educação em saúde é indispensável para o SUS, no cuidado e nas ações de prevenção $e$ promoção à saúde. A estratégia saúde da família é importante para a prática de ações educativas, dado a proximidade com a comunidade. A utilização de tecnologias de informação $e$ comunicação em saúde tem sido ampliado nos últimos anos, com a inserção de ferramentas que dinamizam o fluxo das informações, como as mídias sociais. $O$ objetivo do estudo é evidenciar o uso das ferramentas tecnológicas como suporte para os processos de trabalho na atenção primária à saúde. É uma pesquisa descritiva, do tipo relato de experiência, parte das percepções e vivências em educação em saúde na atenção primária por meio de ferramentas audiovisuais. Os projetos denominados: "Conexão Saúde" $e$ "Hora da Saúde", foram usados para levar informações a comunidade sobre saúde através de áudio e vídeos. Com a pandemia da COVID-19, a atenção primária à saúde teve que adotar medidas estratégicas para continuar ofertando ações de saúde, pois rodas de conversas, palestras e eventos nas unidades básicas de saúde não estavam sendo realizadas. Observouse que os recursos tecnológicos são de grande importância para educação em saúde, permite aprendizagem coletiva e maior interação da equipe de saúde com a comunidade.

Palavras-chave: Educação em saúde. Atenção Primária à Saúde.Acesso a medicamentos essenciais e tecnologias em saúde.Infecções por Coronavírus. 
A educação é definida como uma prática social que implica a utilização de técnicas e processos voltados para a aprendizagem. Torna-se indispensável, no contexto do SUS, especialmente no cuidado e nas ações de prevenção e promoção à saúde. Esse processo denomina-se educação em saúde ${ }^{1}$.

A educação em saúde constitui-se em um processo educativo de apropriação, construção e consolidação de conhecimentos em saúde que contribui para a ampliação da autonomia dos indivíduos e no empoderamento da população a respeito de informações pertinentes $^{2}$.

Pode-se observar, que a utilização de tecnologias de informação e comunicação em saúde tem sido ampliado nos últimos anos, com a inserção de ferramentas que dinamizam o fluxo das informações tais como: email e mídias sociais, que contribuem para a construção do conhecimento em redes e expansão dos canais de comunicação para o acesso livre nos serviços de saúde, sendo um nova estratégia para realizar promoção e prevenção em saúde ${ }^{3}$.

A estratégia saúde da família(ESF), apresenta uma conjuntura privilegiada para a prática de ações educativas, em decorrência da proximidade com a comunidade adscrita, e por ser a equipe de saúde que realiza o primeiro contato com o usuário de forma longitudinal e integral. É o ponto de atenção do SUS com maior complexidade, e que faz uso de tecnologias cognitivas voltadas à prevenção de doenças ${ }^{4}$.

Em março de 2020, o mundo se deparou com um grande desafio no âmbito da saúde, a deflagração da pandemia da COVID-19, declarada pela Organização Mundial da Saúde (OMS). Os primeiros casos do novo coronavírus foram relatados na China, e rapidamente a patologia se disseminou para outros países e continentes devido à alta transmissibilidade ${ }^{5}$.

O quadro clínico dos pacientes acometidos assemelha-se a uma gripe comum, contudo com uma taxa de letalidade maior, 3,4\% em contraste à gripe sazonal que apresenta apenas $0,1 \%$ de letalidade. Os principais sintomas apresentados são: febre, tosse, coriza e em casos mais graves dispnéia. Em decorrência da alta transmissibilidade, foram observadas evidências da necessidade de medidas referentes à quarentena e ao isolamento social, diante da ruptura do tecido social imposta por essas medidas aumentaram o desafio da educação em saúde na atenção primária e sua necessidade ${ }^{6}$.
Este estudo justifica-se pela necessidade de compreender a importância da utilização de recursos audiovisuais no âmbito da atenção básica, servindo como material de apoio e estímulo às práticas de educação em saúde no Sistema Único de Saúde (SUS). O objetivo é evidenciar o uso das ferramentas tecnológicas como suporte para os processos de trabalho na atenção primária.

\section{Metodologia}

Descritivo, do tipo relato de experiência, delineado a partir das percepções e vivências em educação em saúde na atenção primária, por meio de ferramentas audiovisuais. A experiência aconteceu dentro da equipe de estratégia em saúde da família do Baixio das Palmeiras e da Residência Multiprofissional em Saúde Coletiva da Universidade Regional do Cariri (RMSC-URCA), no período de março a agosto de 2020, no CratoCE.

A ESF possui 1.846 usuários cadastrados em todo o seu território, sendo composta por uma equipe mínima de saúde da família e uma equipe de saúde bucal, faz cobertura das unidades básicas de saúde: Baixio das Palmeiras, Baixio do Muquém e Baixio do Romualdo, todos da zona rural.

A experiência refere-se à produção de materiais educativos de promoção à saúde por meio de recursos tecnológicos audiovisuais tais como: podcast e vídeos educativos. Os materiais foram produzidos através de aplicativos online de edição de áudio e vídeo de acesso livre e gratuito. A plataforma utilizada foi o Movie Maker Online que permite aos usuários a criação de mídia social e uma ampla variedade de conteúdos audiovisuais.

As ações de educação em saúde estavam direcionadas para o controle e combate a COVID-19, atualização vacinal, medidas preventivas relacionadas à hipertensão arterial e diabetes, saúde mental e o controle de arboviroses.

\section{Resultados e Discussão}

Diante da pandemia da COVID-19 a utilização de recursos tecnológicos tornou-se essencial frente ao novo contexto vivenciado no ano de 2020. Com a descoberta do novo coronavírus, foram necessárias as mudanças de hábitos para evitar a disseminação dessa doença, 
tais medidas que podemos destacar são: o distanciamento social, o uso de máscaras, higienização das mãos, readequação de ambientes para evitar aglomerações ${ }^{7}$.

Nesta perspectiva, a atenção primária à saúde teve que adotar medidas estratégicas para continuar ofertando as ações de prevenção e promoção à saúde, uma vez que, rodas de conversas, palestras e eventos com grandes aglomerações nas unidades básicas de saúde não estavam sendo realizadas ${ }^{8}$.

Baseado nisso, a equipe de residência multiprofissional em saúde coletiva da URCA, composta pelas seguintes categorias profissionais: enfermagem, farmácia, fisioterapia e nutrição em conjunto com a preceptora e enfermeira da Estratégia Saúde da Família do território Baixio das Palmeiras, desenvolveram dois projetos denominados de "Conexão Saúde" e "Hora da Saúde", com objetivo de levar informações a comunidade com temáticas relacionadas à saúde através de ferramentas de áudio e vídeos.

O projeto "Conexão Saúde" é uma ferramenta de cuidado em saúde no território onde a ESF Baixio das Palmeiras está inserida, a proposta era realizar exibições de vídeos informativos relacionados a temáticasda área da saúde sugeridas pela a comunidade. Enquanto o projeto "Hora da Saúde"utilizava-se de ferramentas de áudios, denominadopodcast.

Em meados do mês de março, com início da pandemia, surgiu à preocupação dos residentes e da preceptora do programa sobre como difundir as informações de medidas preventivas e de educação em saúde sobre o novo coronavírus, uma vez que, as atividades coletivas não eram mais permitidas nas unidades básicas de saúde devido as aglomerações.

A partir disso, a equipe de saúde se dispôs a gravar vídeos educativos, reforçando sobre os cuidados quanto ao distanciamento social, orientações sobre a maneira correta de realizar a higienização das mãos, a importância do uso das máscaras, quais sinais e sintomas que o indivíduo pode apresentar caso seja contaminado pelo o vírus e qual nível de complexidade da rede deve-se procurar de acordo com a gravidade dos sintomas apresentados.

Os vídeos eram gravados na própria unidade de saúde e editados através do aplicativo Movie Maker Online epara que a comunidade tivesse acesso ao material produzido, os residentes desenvolveram um perfil na rede social instagram (@esfbaixiodaspalmeiras) para facilitar o acesso virtual à informação e, além disso, os vídeos também foram compartilhados em grupos de WhatsApp.

Apesar de estarmos vivenciando uma pandemia e as ações estarem direcionadas para o coronavírus, houve a preocupação dos residentes em relaçao as doenças endêmicas da região, visto que, entre o período de março a maio é um período chuvoso na região do cariri e ocorre um aumento nos números de casos de dengue, zika e chikungunya ${ }^{9}$.

A ideia proposta par as doenças endêmicas, foi de desenvolver um vídeo educativo em forma de desenho animado com a caricatura facial dos residentes, cuja finalidade era deixar o vídeo mais atrativo em que crianças e adultos tivessem acesso ao conteúdo e a informação. O material didático trazia os residentes explicando sobre as arboviroses e quais cuidados o usuário deveria adotar em sua residência para prevenir a disseminação e propagação da doença.

Outro vídeo produzido pela a equipe e mediado pela a nutricionista residente foi sobre dicas de como melhorar a alimentação em tempos de pandemia. A alimentação tem um papel relevante na saúde das pessoas, na qual alimentos saudáveis e de uma ampla diversidade de consumo são capazes de potencializar as ações do nosso sistema imunológico, mas cabe ressaltar que a alimentação por si só, não é uma garantia de ficar imune a COVID-19 ${ }^{10}$.

Em relação às ferramentas de áudio em formato podcast, o mesmo era exibido na plataforma Soundcloud, esta ferramenta é uma das maiores plataforma de áudio aberta do mundo, o link do podcast era compartilhado nas redes sociais para que a comunidade tivesse acesso ao conteúdo.

Além disso, o áudio do podcast também era executado nos alto-falantes das igrejas da comunidade e em carros de som, para que ocorresse a divulgação da informação para pessoas de vulnerabilidade social e sem acesso a internet.

Quanto aos eixos temáticos dos podcast o enfoque foi para as medidas de controle, combate e prevenção da COVID-19, e sobre os cuidados com a saúde mental em tempos de pandemia. Vale salientar, que devido ao avanço da doença, o isolamento social e o excesso de informações disponíveis na mídia, tornam-se um meio propício para o adoecimento psicológico, por isso é de fundamental importância a implementação de estratégias dentro do território de ações educativas relacionada à saúde mental ${ }^{11}$. 
Dessa forma, ressalta-se que ferramentas da Web 2.0 são de grande relevância para educação em saúde, na qual podemos citar alguns exemplos: os Blogs, wikis, podcast e redes sociais. Estes recursos têm sido cada vez mais utilizados por profissionais da saúde, devido à facilidade de manuseio e implantação, oferecendo assim agilidade na partilha de informações para usuários ${ }^{12}$.

Outro projeto articulado pela a equipe foi à rádio comunitária, que tinha como propósito levar informações sobre saúde para a comunidade através dos alto-falantes das igrejas. A ideia proposta era reforçar sobre campanhas vacinais e educação em saúde relacionada à hipertensão e diabetes, doenças com grande predominância de casos no território. As ações de educação em saúde eram transmitidas em tempo real e mediada pelos os residentes.

Esta necessidade de inserir novas ferramentas tecnológicas no âmbito do SUS surgiu em 2007. O Ministério da Saúde institui o programa Nacional de Telessaúde Brasil em redes através da portaria no 35 e posteriormente é expandido através de uma nova portaria de no 2.546 em outubro de 2011 que define e especifica ferramentas tecnológicas de informação e comunicação ${ }^{13}$. Essas ferramentas tecnológicas ganharam vigor em decorrência da pandemia, fruto do isolamento e distanciamento social, onde as pessoas tiveram que buscar novas estratégias e se adequar à nova realidade.

A educação em saúde a distância deve ser compreendida como um novo espaço aberto de participação ativa da comunidade, em condições de total igualdade de acesso à informação. Promover saúde com auxílio de ferramentas tecnológicas é um novo paradigma de organização social de um vasto campo de conhecimentos, permitindo o acesso a materiais educativos em saúde e consequentemente a informação ${ }^{14}$.

\section{Considerações finais}

Esse estudo discorreu sobre o uso de ferramentas audiovisuais para educação em saúde na atenção primária. Foi possível observar que os recursos tecnológicos são de grande importância para educação em saúde, pois permite uma aprendizagem coletiva promovendo uma maior interação da equipe de saúde com a comunidade.

Os meios de comunicação audiovisuais foi uma estratégia articulada pela a equipe de saúde da família e da residência multiprofissional em saúde coletiva da URCA, como uma forma de promover informações e garantir em tempos de pandemia, o distanciamento social e o acesso às informações, especificamente para populações mais vulneráveis localizadas em locais de difícil acessibilidade.

A literatura reforça que o uso de mídias digitais começou a ser utilizado no ano de 2007, indicando que o uso dessas ferramentas para educação em saúde ainda está em processo de construção, isso deixa uma caminho para a comunidade científica a investigar mais sobre a temática e novas possibilidades de inserir estes recursos nos serviços de saúde.

\section{Referências}

1. Almeida Edmar Rocha, Moutinho Cinara Botelho, Leite Maisa Tavares de Souza. A prática da educação em saúde na percepção dos usuários hipertensos e diabéticos. Saúde debate [Internet]. 2014 June [cited 2021 Apr 08] ; 38( 101 ): 328-337. Available from: http://www.scielo.br/scielo.php?script=sci_artte $\mathrm{xt} \& \mathrm{pid}=\mathrm{S0103}$ -

$11042014000200328 \&$ Ing=en. http://dx.doi.org/ 10.5935/0103-1104.20140030.

2. Ceccon Roger Flores, Schneider Ione Jayce Ceola. Tecnologias leve em tempos de pandemia: A educação em saúde como dispositivo de combate ao coronavírus. Scientific Electronic Library Online.Disponivel: https://preprints.scielo.org/index.php/scielo/pre print/view/136/160.

3. Pinto Luiz Felipe, Rocha Cristianne Maria Famer. Inovações na Atenção Primária em Saúde: o uso de ferramentas de tecnologia de comunicação e informação para apoio à gestão local. Ciênc.saúdecoletiva [Internet]. 2016 May [cited 2021 Apr 08] ; 21( 5 ): 1433-1448. Available from: http://www.scielo.br/scielo.php?script=sci_artte xt\&pid=S1413-

$81232016000501433 \&$ Ing=en. https://doi.org/10 .1590/1413-81232015215.26662015.

4. Rios AFM, Lira LSSP, Reis LM, Silva GA. Atenção primária à saúde frente à Covid-19 em um centro de saúde. Enferm. foco (Brasília). [Internet]. 2020[acesso em 2020 out 24];11(1):246-51. Disponível em: http://revista.cofen.gov.br/index.php /enfermagem/article/download/3666 /836.

5. Ceccon Roger Flores, Schneider Ione Jayce Ceola. Tecnologias leve em tempos de pandemia: A educação em saúde como dispositivo de combate ao coronavírus. Scientific 
Electronic Library Online.Disponivel: https://preprints.scielo.org/index.php/scielo/pre print/view/136/.

6. Sarti Thiago Dias, Lazarini Welington Serra, Fontenelle Leonardo Ferreira, Almeida Ana Paula Santana Coelho. Qual o papel da Atenção Primária à Saúde diante da pandemia provocada pela COVID-19?. Epidemiol. Serv. Saúde [Internet]. 2020 [cited 2021 Apr 08] ; 29( 2 ): e2020166. Available from: http://www.scielo.br/scielo.php?script=sci_artte $x t \& p i d=S 2237$ -

96222020000200903\&lng=en. Epub Apr 27, 2020. https://doi.org/10.5123/s167949742020000200024

7. Daumas Regina Paiva, Silva Gulnar Azevedo e, Tasca Renato, Leite luri da Costa, Brasil Patrícia, Greco Dirceu B. et al . O papel da atenção primária na rede de atenção à saúde no Brasil: limites e possibilidades no enfrentamento da COVID-19. Cad. Saúde Pública [Internet]. 2020 [cited 2021 Apr 08] ; 36( 6 ): e00104120. Available

from: http://www.scielo.br/scielo.php?script=sci_artte $x t \& p i d=S 0102$

311X2020000600503\&Ing=en. Epub June 26, 2020. https://doi.org/10.1590/0102-

\section{1x00104120.}

8. Lima-Camara Tamara Nunes. Arboviroses emergentes e novos desafios para a saúde pública no Brasil. Rev. Saúde Pública [Internet]. 2016 [cited 2021 Apr 08] ; 50: 36. Available from: http://www.scielo.br/scielo.php?script=sci_artte

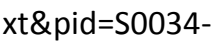

89102016000100602\&lng=en. Epub June 27, $2016 . \quad$ http://dx.doi.org/10.1590/S15188787.2016050006791.

9. Paixão de Gois B, Dias Pereira A, Lays Soares Lopes K, Campos Corgosinho F. Suplementação e alimentação adequada no contexto atual da pandemia causada pela covid19. Driuft [Internet]. $22 \circ$ de abril de 2020 [citado 8ㅇ de abril de 2021];7(Especial-3):89-6. Disponível em: https://sistemas.uft.edu.br/periodicos/index.php /desafios/article/view/8825.

10. Pereira MD, Oliveira LC de, Costa CFT, Bezerra CM de O, Pereira MD, Santos CKA dos, Dantas EHM. A pandemia COVID-19, isolamento social, consequências na saúde mental e estratégias de enfrentamento: uma revisão integrativa. RSD [Internet]. 5 de junho de 2020 [citado em 8 de abril de 2021]; 9 (7): e652974548. Disponível em: https://rsdjournal.org/index.php/rsd/article/view /4548.
11. Cruz DI, Paulo RRD, Dias WS, Martins VF, Gandolf PE. O uso das mídias digitais na educação em saúde. Cad FUCAMP. 2011;10(13):130-42.

12. Brasil. Ministério da Saúde. Portaria no 1.399, de 15 de dezembro de 1999. 2.546, de 27 de Outubro de 2011. Redefine e amplia o Programa Telessaúde Brasil, que passa a ser denominado Programa Nacional Telessaúde Brasil Redes (Telessaúde Brasil Redes). Diário Oficial da União. 27 Out 2011.

13. Schall Virgínia Torres, Modena Celina Maria. As novas tecnologias de informação e comunicação em educação em saúde. In: Minayo MCS, Coimbra JR, cea, organizadores. Críticas e atuantes: ciências sociais e humanas em saúde na América Latina [online]. Rio de Janeiro: Cebes; 2009. p. 24-46.

\section{Endereço para Correspondência}

Antonio Rafael da Silva

Rua Ratisbona no 32 Apt 302 - Centro.

Crato-CE - Brasil

CEP: $63100-140$

E-mail: raphaelsilvha@gmail.com

Recebido em 16/04/2021

Aprovado em 01/06/2021

Publicado em 30/12/2021 